\title{
Modelling Signal Controlled Traffic Based on Driving Behaviors
}

\author{
Yang Wang, Yanyan Chen, and Ning Chen \\ Beijing Key Lab of Traffic Engineering, College of Metropolitan Transportation, Beijing University of Technology, Beijing 100124, China \\ Correspondence should be addressed to Yanyan Chen; cdyan@bjut.edu.cn
}

Received 13 October 2014; Revised 26 December 2014; Accepted 7 January 2015

Academic Editor: Carlo Piccardi

Copyright (C) 2015 Yang Wang et al. This is an open access article distributed under the Creative Commons Attribution License, which permits unrestricted use, distribution, and reproduction in any medium, provided the original work is properly cited.

\begin{abstract}
In urban traffic, of particular interest the traffic breakdown which is primarily resulted from the driving behaviors is emerged to respond to the traffic signal. To investigate the influences of driving behaviors on the traffic breakdown, a cellular automaton model has been developed by incorporating a number of driving behaviors typically manifesting during the different stages when the vehicle approaching a traffic light. Numerical simulations have been performed based on a road segment consisting of three sections and each section is associated with a set of rules. The numerical simulations have demonstrated that the proposed model is capable of producing the time-delayed traffic breakdown and the dissolution of the oversaturated traffic. Furthermore, it has been evidenced that the probability of the traffic breakdown can be increased by involving the slow-to-start behavior. However, the activation of the anticipatory behavior can effectively impede the transition from undersaturated to oversaturated traffic. Finally, the contributions of the driving behaviors on the traffic breakdown have been quantitatively examined.
\end{abstract}

\section{Introduction}

To study the traffic problems, many researchers in physics, transportation, and mathematics communities have proposed various models based on cellular automaton (CA). CA is one of useful tools to reproduce some traffic phenomena based on a set of rules which are used to describe the interactions between neighboring vehicles. Therefore, CA models for the traffic flow have become more fruitful, since the seminal work of Nagel and Schreckenberg [1].

The NaSch model proposed by Nagel and Schreckenberg [1] is generally considered to be "minimal" to reproduce the basic features of the traffic flow (e.g., spontaneous formation of jams), but even so it is far from realistic as it incorporates few driving behaviors. In this respect, a considerable number of cellular automaton models have been developed to improve and extend the NaSch model, by incorporating aggressive acceleration [2], velocity-dependent randomization [3], comfortable driving [4], anticipation behavior [5], brake light effect [6-9], speed difference dependent randomization [10], time gap dependent randomization [11], defensive reaction [12], and so forth. An excellent review can be found in [13].

The existing CA models for the signal controlled traffic flow can be classified into two categories: one category focuses on the interaction between traffic flows from different directions at a signalized intersection based on two-dimensional traffic modelling and the other attempts to reproduce the traffic phenomena induced by the traffic signal using onedimensional cellular automata. As the CA model proposed in this paper aims to incorporate driver's reactions to the traffic light, one-dimensional CA is adopted and consequently related works are briefly summarized here. D. W. Huang and W. N. Huang employed the NaSch model to investigate the synchronization effects for a set of traffic signals and found that the benefits of synchronization are obvious when the traffic density is low but marginal when traffic demand surpasses the saturated flow [14]. Neumann and Wagner modified the NaSch model by introducing deceleration probability and analyzed the impact of traffic lights on travel time [15]. Jiang and $\mathrm{Wu}$ introduced a stopped time dependent randomization mechanism into the original $\mathrm{NaSch}$ model for the signal controlled intersection [16]. Varas et al. examined two control strategies for a sequence of traffic lights with a simple CA in which the velocity of vehicles is limited to two values of 0 and 1 [17]. In [18], the NaSch model was used to investigate the effects of four information feedback strategies on a scenario where two traffic lights were introduced to regulate the entrance to an overlapped road link for the vehicles from two origins. de Gier et al. developed a CA 
TABLE 1: Typical driving behaviors and corresponding velocity update rules for the vehicles on the effective section.

\begin{tabular}{lll}
\hline Phase & Moving $\left(v_{i}(t)>0\right)$ & Stopped $\left(v_{i}(t)=0\right)$ \\
\hline & Moving as fast as possible & Slow-to-start at the beginning \\
& GM.R1: $v_{i}^{\prime}(t)=\min \left\{v_{i}(t)+a, v_{\max }^{\text {eff }}\right\}$ & GS.R1: $v_{i}^{\prime}(t)=\min \left\{v_{i}(t)+1, v_{\max }^{\text {eff }}\right\}$ \\
Green & GS.R2: $v_{i}^{\prime \prime}(t)=\min \left\{v_{i}^{\prime}(t), d_{i}(t)\right\} ;$ \\
& $d_{i}(t)=g_{i}(t)+b_{i-1}(t) v_{i-1}(t)$ & $d_{i}(t)=g_{i}(t)+b_{i-1}(t) v_{i-1}(t)$ \\
& GM.R3: $v_{i}(t+1)=\max \left\{v_{i}^{\prime \prime}(t)-1,0\right\}$ with $p_{g m}$ & GS.R3: $v_{i}(t+1)=\max \left\{v_{i}^{\prime \prime}(t)-1,0\right\}$ with \\
& Slowdown and even $\operatorname{stop}$ & $p_{g s}=\min \left\{p_{g} \max , p_{g} \min \right.$ \\
& RM.R1: $v_{i}^{\prime}(t)=\min \left\{v_{i}(t)+a, v_{\max }^{\text {eff }}\right\}$ & Inch-forward to reduce the distance \\
\hline \multirow{4}{*}{ Red } & RM.R2: $v_{i}^{\prime \prime}(t)=\min \left\{v_{i}^{\prime}(t), d_{i}(t)\right\} ;$ & RS.R1: $v_{i}^{\prime}(t)=\min \left\{v_{i}(t)+1, v_{\max }^{\text {eff }}\right\}$ \\
& $d_{i}(t)=\min \left\{g_{i}(t)+b_{i-1}(t) v_{i-1}(t), G(t)\right\}$ & RS.R2: $v_{i}^{\prime \prime}(t)=\min \left\{v_{i}^{\prime}(t), d_{i}(t)\right\} ;$ \\
& RM.R3: $v_{i}(t+1)=\max \left\{v_{i}^{\prime \prime}(t)-a, 0\right\}$ with $p_{r m}$ & $d_{i}(t)=\min \left\{g_{i}(t)+b_{i-1}(t) v_{i-1}(t), G(t)\right\}$ \\
& & RS.R3: $v_{i}(t+1)=\max \left\{v_{i}^{\prime \prime}(t)-1,0\right\}$ with $p_{r s}$ \\
\hline
\end{tabular}

model of for generic urban road networks and applied this model to compare the effects of nonadaptive versus adaptive traffic signals for the vehicles obeying NaSch dynamics [19]. Chowdhury and Schadschneider developed a CA model for traffic flows in cities by combining the Biham-MiddletonLevine (BML) model and the NaSch model [20]. They attempted to present the intersections between signal and vehicle and between vehicles by modifying the deceleration mechanism of the original NaSch model, but other driving behaviors (i.e., the behaviors listed in Table 1) are not taken into account.

Based on the above review, it is obvious that the previous works have hardly focused on modelling the driving behaviors in response to the traffic light, but the most of previous works adopted the NaSch model (or similar models) to exam traffic flows when various traffic signal control strategies were applied. As a result, some important phenomenon may remain undercover due to the lack of adequate modelling for the signal-vehicle interaction. Also, little is known about which microscopic behavior is responsible for a specific macroscopic traffic phenomenon. Furthermore, driving behaviors are rather different for the moving and stopped vehicles during different signal phases, which are not separated in the previous works.

In an attempt to remedy the aforesaid shortcomings, this paper proposes a CA model by incorporating the driving behaviors typically observed for the vehicles approaching a traffic signal. The traffic scenario and the developed model and traffic scenarios will be introduced in the next section. Then, the primary exploration will be presented and the obtained results discussed in Section 3. The paper is concluded in Section 4.

\section{The Traffic Scenario and the Proposed Model}

2.1. The Signalized Traffic Scenario. With the aim to model the driving behaviors induced by the traffic signal, this research work adopts a one-dimensional lattice to construct a single lane road segment and all vehicles are assumed to move from the left to the right, as shown in Figure 1. The previous studies [21, 22] have proved that it is sufficient to consider traffic breakdown at a single signalized intersection in order

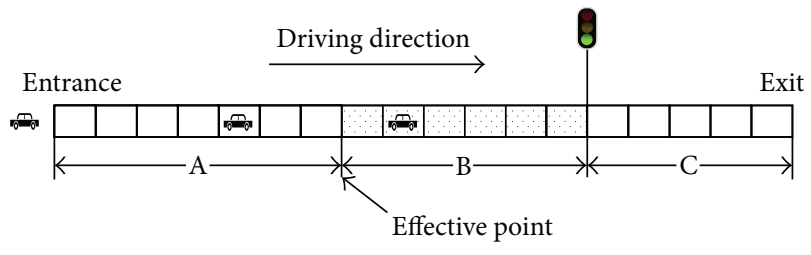

FIGURE 1: The signal controlled traffic scenario.

to reveal features of traffic breakdown at the signal. Therefore, a traffic light which operates in a fixed cycle manner is amounted on the single lane road segment. Furthermore, unlike the previous works, the road segment is split into three sections, that is, A, B, and C, and different rules have been developed for each section. Section A represents the part on which the vehicular movement is not significantly affected by the traffic light possibly due to the relatively long distance. When the vehicles pass the effective point, the influence of the traffic light needs to be considered in order to avoid the traffic violation and collision. Sections B and C are separated by the traffic light, as the interaction between the vehicles is dominated after the vehicles pass the traffic light. All road sections are subdivided into a number of cells with the same length. Note that the amber light is omitted in this paper for the reason that the microscopic vehicular behavior has little influence on the traffic dynamics due to the short period and therefore the infinite deceleration mechanism is adopted to ensure a movement without traffic violation. Furthermore, this paper assumes that the maximum permitted velocity $v_{\max }^{\text {eff }}$ for the effective section is smaller than that $v_{\max }^{\text {nor }}$ for the normal sections, as the driving speed is often restricted in intersections to prevent vicious accidents between vehicles and pedestrians. For simplicity, one type of vehicles and homogeneous drivers are assumed in the following.

2.2. The Proposed CA Model. Two sets of rules have been developed to account for the different behaviors for the vehicles moving on the effective and normal sections. Before explaining the rules, the definitions on the variables and parameters used in the model are given as follows.

Let $T_{g}, T_{r}$, and $T_{a}$ be the lengths of green, red, and amber phase, respectively, and $\bar{t}_{g}, \bar{t}_{r}$, and $\bar{t}_{a}$ be the remaining time for 
each phase. $\tau_{i}(t)$ is the amount of time for which the vehicle $i$ has been stopped until the time step $t . x_{i}(t)$ and $v_{i}(t)$ denote the position and velocity, respectively, of vehicle $i$, and $x_{i-1}(t)$ and $v_{i-1}(t)$ are the position and velocity, respectively, of the vehicle ahead, at the time step $t$. In this paper, the vehicle is allowed to accelerate at a rate $a(a>1)$ rather than 1 in the NaSch model [1]. $b_{i}$ is the status of the brake light of vehicle $i$, with 0 for on and 1 for off. While the gap from the vehicle $i$ to the preceding vehicle $i-1$ at the time step $t$ is $g_{i}(t)=x_{i-1}(t)-x_{i}(t)-l_{i-1}$ (where $l_{i-1}$ is the length of vehicle $i-1), G_{i}(t)$ denotes the distance from its current location to the stop line (i.e., the same position as the traffic light in this paper). $h$ is a distance threshold value and the driver's reaction is effectively affected by the brake light of the preceding vehicle if $g_{i}(t) \leq h . p_{g m}, p_{g s}, p_{r m}$, and $p_{r s}$, denote the probability to be taken for the random deceleration for the moving and stopped vehicles during green and red phases, respectively. While $p_{g \text { max }}$ and $p_{g \text { min }}$ denote the maximum and minimum values for $p_{g s}$ for the effective section, the maximum and minimum probabilities of randomization for a vehicle stopped at the normal sections are $p_{n \text { max }}$ and $p_{n \text { min }}$, respectively. The scaling factors $\theta_{g}$ and $\theta_{n}$ will be explained in the following.

(1) Rules for the Effective Section. Table 1 briefly summarizes the typical driving behaviors generally observed in reality for the vehicles and corresponding rules developed for green and red phase.

For the green phase, three rules have been designed for the velocity update so as to reflect the fast movement for the moving vehicles. First of all, all moving vehicles on the effective section are subject to a process of acceleration, implying the intention of drivers to pass the intersection as fast as possible. Knowledge of the preceding vehicle's velocity $v_{i-1}(t)$ at the time step $t$ is incorporated into the safe distance $d_{i}(t)=g_{i}(t)+b_{i-1}(t) v_{i-1}(t)$, provided that it is close to its preceding vehicle (i.e., $g_{i}(t) \leq h$ ). In another word, the movement of the preceding vehicles has been preestimated by the means of the brake light and considered when calculating the safe distance, to reflect the anticipatory behavior. The anticipatory behavior reported in [5] assumes that the velocity of the preceding vehicle can be estimated, but an extra parameter was introduced to control the degree of estimation accuracy. The other works [6-9] claimed that the velocity of the preceding vehicle can be anticipated from the gap $g_{i-1}$ between the preceding vehicle and the vehicle ahead. This implies that the preceding vehicle follows the same rule to move. Furthermore, the complete knowledge about the gap $g_{i-1}$ is obviously unrealistic. Based on these two considerations, we have improved the anticipatory behavior by reference to the traffic practice. It has been evidenced that driver's perception of speed change of an object in motion is very inaccurate unless it is observed for a relatively long time (i.e., $10 \mathrm{~s}$ and more) [23]. For this reason, in the proposed model, the preceding vehicle is conservatively assumed to move at the constant speed when its brake light is off. On the other hand, it is rather safe to assume that the preceding vehicle brakes hard until the preceding vehicle comes a complete stop if the brake light of the preceding vehicle is on. The anticipatory behavior can result in a small gap between the successive vehicles, thus yielding a closely following pattern. However, it should be noted that this paper assumes that the driver can accurately estimate the previous velocity of the preceding vehicle and it is often valid for an experienced driver. In addition, it has been found that many drivers decelerate without any exogenous reasons on highway [1]. This may not be common for the signalized traffic, as more drivers would concentrate on driving when approaching an intersection where traffic conflicts are likely to be more. Thus, a small probability should be assigned to the random deceleration (i.e., GM.R3). Note that only small deceleration rate of 1 is allowed in GM.R3 to reflect the fast movement.

It is quite common that drivers lose their attention to the signal while waiting at signalized intersections and may initiate other activities (e.g., making a call, playing with mobile phone, reading newspapers, and cleaning up windows), potentially resulting in a slow-to-start $[24,25]$ when the light changes to green. Thus, this slow reaction is a typical behavior that the proposed model attempts to incorporate for a stopped vehicle. The first two rules are the same as those for the moving vehicles during green phase, except that only a small acceleration rate of 1 is allowed for the stopped vehicle to reflect the slow reaction. However, the random deceleration with probability $p_{g s}$ will eventually make a proportion of waiting vehicles to remain stopped after the light changes to green. This has been modelled by linearly scaling the stopped time by a constant $\theta_{g}$, as that in [26]. The probability $p_{g s}$ should be larger than $p_{g m}$, because a stopped vehicle generally takes more time to accelerate than a moving one does due to the inertia of the vehicle or the reaction delay [24]. Furthermore, it should be aware that $p_{g \text { min }}$ is equal to $p_{g m}$ in fact.

Although the rules for the moving vehicles during the red phase are largely similar to those during the green phase, the distance to the stop line $G(t)$ is taken into account when calculating the safe distance. More specifically, the vehicle will come to the stop line, if the distance to the stop line $G(t)$ is smaller than the distance to its preceding vehicle (i.e., $g_{i}(t)+b_{i-1}(t) v_{i-1}(t)$ as explained above) and vice versa. Subsequently, a proportion of the moving vehicles are subject to a further deceleration process. This is achieved by applying a large deceleration rate (equal to the acceleration rate). The number of vehicles that randomly slowdown can be controlled by the probability $p_{r m}$. That is, the larger the probability $p_{r m}$ is, the fewer the vehicles will speed up.

During the red phase, if a vehicle is stopped far from the stop line or its preceding vehicle, the following three rules can help it inch forward to reduce the gap to the stop line or its preceding vehicle. First of all, all vehicles are intended to accelerate at the smallest rate. The second rule reflects that such acceleration is not feasible if a traffic violation or collision risk exists. The last rule implies that a number of drivers would not like to move forward to reduce the gap possibility due to their laziness.

A simple rule has been developed for the moving vehicles during the amber phase. That is, if a vehicle can pass the traffic light at its current velocity, it will follow the rules developed for the green phase, and, otherwise, the rules developed for the red phase will be employed. On the other hand, any 
stopped vehicles will remain stationary during the amber phase.

To unify the rules stated above, one extra variable $f$ is introduced to indicate the signal phase, with 1, 0, and - 1 being green, amber, and red, respectively. Finally, an additional rule is appended to the unified three rules for the motion update. These four rules are used consecutively to update the motions of all vehicles on the effective section.

E.R1: Acceleration. One has $v_{i}^{\prime}(t)=\min \left\{v_{i}(t)+a, v_{\max }^{\text {eff }}\right\}$ with

$$
\begin{gathered}
\quad a>1, \quad \text { if } v_{i}(t)>0 \\
a=1, \quad \text { if } f=-1, v_{i}(t)=0 \\
a=0, \quad \text { if } f=0, v_{i}(t)=0 .
\end{gathered}
$$

E.R2: Deceleration. One has $v_{i}^{\prime \prime}(t)=\min \left\{v_{i}^{\prime}(t), d_{i}(t)\right\}$;

$$
d_{i}(t)
$$$$
=\left\{\begin{array}{l}
g_{i}(t)+b_{i-1}(t) v_{i-1}(t), \\
\text { if }(f=1) \text { or }\left(f=0, v_{i-1}(t)>0, \frac{G(t)}{v_{i-1}(t)}<\bar{t}_{a}\right) \\
\min \left\{g_{i}(t)+b_{i-1}(t) v_{i-1}(t), G(t)\right\}, \\
\text { otherwise. }
\end{array}\right.
$$

E.R3: Randomization. One has $v_{i}(t+1)=\max \left\{v_{i}^{\prime \prime}(t)-\right.$ $b, 0\}$ with

$p$

$$
= \begin{cases}p_{g m}, & \text { if } f=1, v_{i}(t)>0 \\ p_{g s}=\min \left\{p_{g \text { max }}, p_{g \text { min }}+\theta_{g} \tau_{i}\right\}, & \\ p_{r m}, & \text { if } f=1, v_{i}(t)=0 \\ p_{r s}, & \text { if } f=0, v_{i}(t)>0 \\ \text { if } f=0, v_{i}(t)=0,\end{cases}
$$

$b= \begin{cases}a, & \text { if }\left(f=-1, v_{i}(t)>0\right) \text { or }\left(f=0, \frac{G(t)}{v_{i-1}(t)} \geq \bar{t}_{a}\right) \\ 1, & \text { otherwise. }\end{cases}$

$$
\text { E.R4: Motion. } x_{i}(t+1) \rightarrow x_{i}(t)+v_{i}(t+1) \text {. }
$$

(2) Rules for the Normal Sections. The rules for the normal sections are similar to the rules for the vehicles on the effective section during green light. However, the anticipatory behavior is excluded so as to reflect the reactive behavior for the normal sections. Furthermore, unlike the rules for the effective section, the deceleration rate in N.R3 is set the same as the acceleration rate. The slow-to-start behavior is also embedded by assuming that the probability for randomization is proportional to the stopped time with a scaling factor $\theta_{n}$. At each discrete time step, all the vehicles on the normal sections are subject to a simultaneous motion update according to the following rules.
N.R1: Acceleration. One has $v_{i}^{\prime}(t)=\min \left\{v_{i}(t)+a\right.$, $\left.v_{\max }^{\text {nor }}\right\}$.

N.R2: Deceleration. One has $v_{i}^{\prime \prime}(t)=\min \left\{v_{i}^{\prime}(t), g_{i}(t)\right\}$.

N.R3: Randomization. One has $v_{i}(t+1)=\max \left\{v_{i}^{\prime \prime}(t)-\right.$ $a, 0\}$ with $p=\min \left\{p_{n \text { max }}, p_{n \text { min }}+\theta_{n} \tau_{i}\right\}$.

N.R4: Motion. One has $x_{i}(t+1) \rightarrow x_{i}(t)+v_{i}(t+1)$.

\section{Numerical Experiments}

Numerical simulations have been performed with the aim to identify the traffic phenomenon produced by the model and the driving behaviors responsible for a specific phenomenon. Before presenting the results, the experiment settings are described firstly.

All the simulations have been performed on a road segment which consists of a single lane of 500 cells with each cell corresponding to $2 \mathrm{~m}$. All vehicles are assumed to be $6 \mathrm{~m}$ in length and a vehicle occupies 3 cells equivalently. A traffic light is located at the 301 cell from the entrance and the effective section is 100 cells (equal to $200 \mathrm{~m}$ ) in length. The road is initially assumed to be empty. Vehicles with maximum velocity enter into the system at a constant rate of 720 vehicles/h. The maximum velocity has been set to be 9 cells/step (corresponding to $64.8 \mathrm{Km} / \mathrm{h}$ ) for the normal sections. As we have frequently observed that the majority of the vehicles approaching a traffic light slow down in order to better manage the unexpected and emergent incidents but a few drivers pass without deceleration in our city, we set 9 cells/step and 5 cells/step (corresponding to $64.8 \mathrm{Km} / \mathrm{h}$ and $36 \mathrm{Km} / \mathrm{h}$ ) as the maximum velocity for the vehicles driving on the effective section with probabilities 0.1 and 0.9 , respectively. While the acceleration rate is assumed to be $4 \mathrm{~m} / \mathrm{s}^{2}$ for the moving vehicles, the stopped vehicles can only start at the rate of $2 \mathrm{~m} / \mathrm{s}^{2}$ to reflect the inertia of the vehicle. $80 \mathrm{~s}$ has been chosen for the signal cycle length, with $T_{g}=27 \mathrm{~s}, T_{r}=50 \mathrm{~s}$, and $T_{a}=3 \mathrm{~s}$, respectively. The reason for choosing such fixed timing is that the examination on the signal timing is beyond the scope of this paper. On the normal road sections, $p_{n \text { max }}$, $p_{n \text { min }}$, and $\theta_{n}$ have been set as $0.1,0.3$, and 0.01 , respectively, in all simulations. Additionally, the threshold $h$ for the reaction range of brake light is 10 cells (equal to $20 \mathrm{~m}$ ).

3.1. The Time-Delayed Traffic Breakdown. The first set of simulations were performed without the activation of anticipatory behavior under the parameter setting as $p_{g m}=0.1$, $p_{\text {max }}=0.6, \theta_{g}=0.01, p_{r m}=0$, and $p_{r s}=0$. Figure 2 shows the typical spatiotemporal pattern for the traffic flows generated from two independent realizations. It is clear that the traffics transit from the initial undersaturated state into the oversaturated state after a time delay of the 1360 time step (the 17 cycle) and the 2560 time step (the 32 cycle) approximately, as shown in Figures 2(a) and 2(b), respectively. Furthermore, after performing a number of independent runs, it has been found that the time delay for the traffic breakdown is random. This finding is consistent with the phenomenon recently reported in $[22,26]$. 


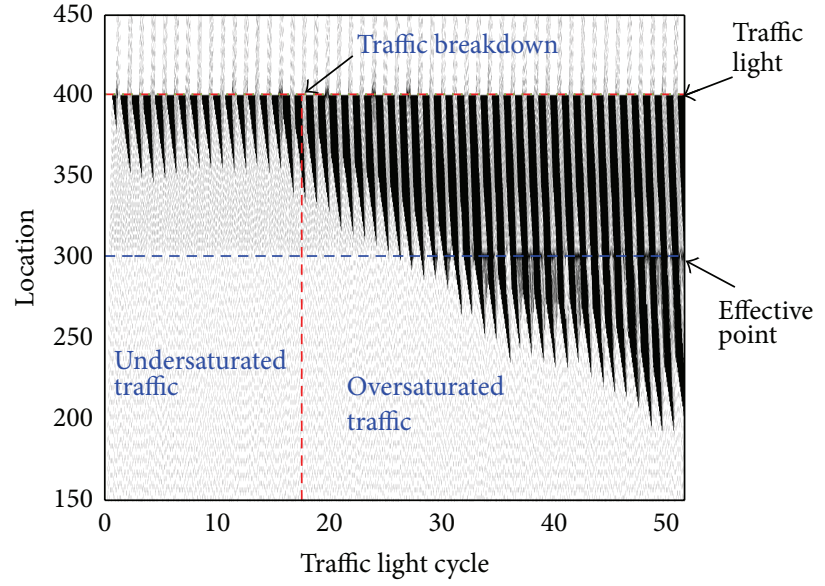

(a)

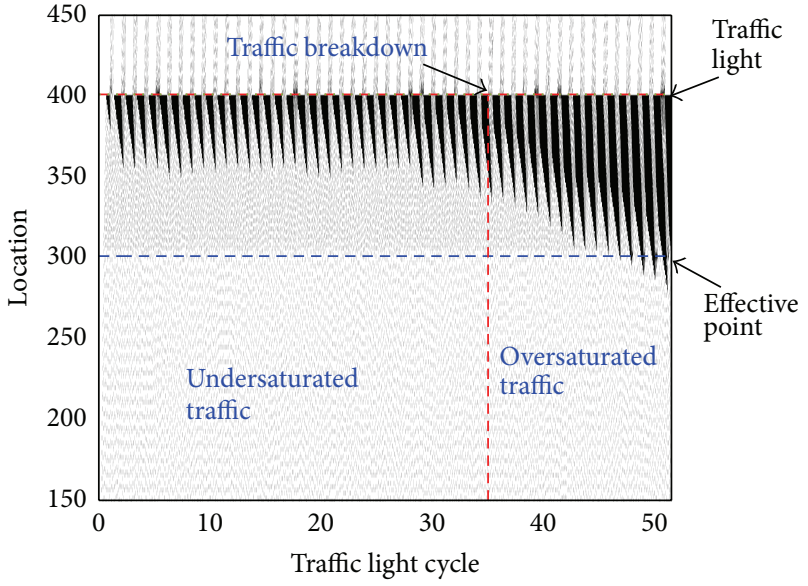

(b)

FIGURE 2: The spontaneous traffic breakdown after a time delay, one realization is shown in (a) and another in (b).

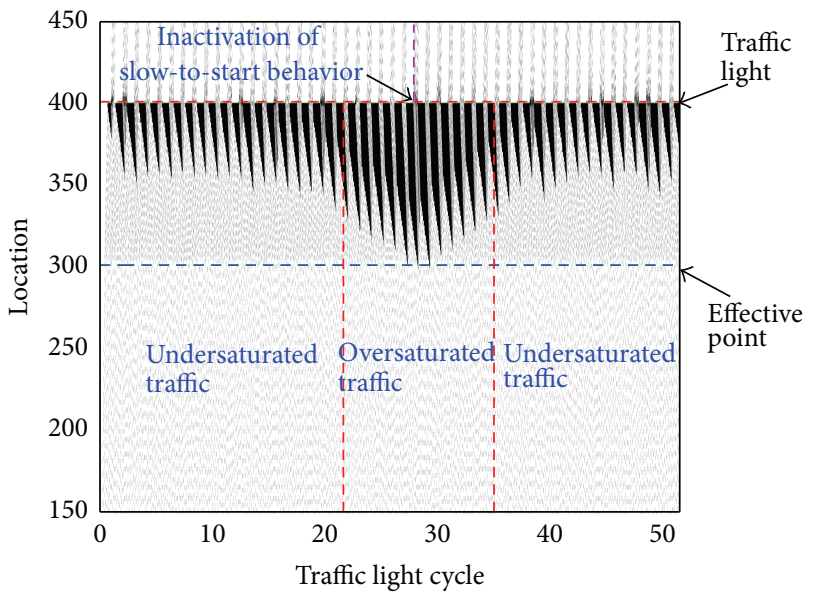

FIGURE 3: Traffic evolution when the slow-to-start behavior was initially activated and then inactivated after the oversaturation lasted for five cycles.

Figure 3 shows that the traffic firstly experienced a transition at 21 cycles from the undersaturation to the oversaturation when the slow-to-start behavior was activated and a reversed transition at 35 cycles when the slow-to-start behavior was inactivated after the oversaturated traffic lasted for five cycles. Note that the inactivation of the slow-tostart behavior was realized by setting $\theta_{g}=0$ and the other parameters were kept as above. The two transitions shown in Figure 3 indicate that the slow-to-start behavior is responsible for the traffic breakdown.

Two virtual detectors have been used to collect the data at the effective point and the traffic light in order to estimate relevant parameters. Figures 4 to 7 show the results averaged over 100 independent runs with the traffic breakdown at the 20 cycle approximately.

Figure 4 presents the distributions of the number of vehicles passing the effective point and the traffic light during each cycle. An increase and a slight decrease after the traffic breakdown can be observed from Figures 4(a) and 4(b), respectively. These patterns will be discussed later in conjunction with the results presented in Figures 5 and 6 . However, before the traffic breakdown, the numbers of vehicles passing the effective point are smaller than those passing the traffic light.

The results presented in Figures 5(a) and 5(b) are the speeds averaged for each cycle when the vehicles passing the effective point and the traffic light. In Figure 5(a), it can be seen that there is a big drop in the time mean speed when the oversaturated traffic continues for several cycles since the traffic breakdown. This drop will be discussed later with the time headway presented in Figure 6(a). However, there is no significant difference in the time mean speed before and after the traffic breakdown, as shown in Figure 5(b).

Figure 6 presents the time headway averaged for each cycle when the vehicles crossing the effective point and the traffic light. Note that the time headway will not be included if a vehicle cannot cross the effective point or the traffic light during the current cycle. In Figure 6(a), the averaged time headways vary around 4 before the traffic breakdown, but a decrease can be observed after the 26 cycles approximately and the time headway becomes relatively small and stable after the 40 cycles around. On the other hand, the time headway in Figure 6(b) varies slightly over the test period as compared to that in Figure 6(a). However, by a close observation, there is an increase during the period around the breakdown cycle, as shown in the figure inserted in Figure 6(b).

By comparing Figures 4(a), 5(a), and 6(a), it can be found that the big changes start from the 26 cycle around and terminate before the 40 cycles. After checking the simulations, we have found that the upstream of the queue was extended gradually into Section A and the transient process starts from the 26 cycles and ends at the 40 cycles approximately. The lower the speed of a vehicle is, the shorter the distance to the preceding vehicle is and therefore the larger the number of vehicles crossing the effective points is. In contrast, the number of vehicle passing the traffic light becomes less after the traffic breakdown, as shown in 


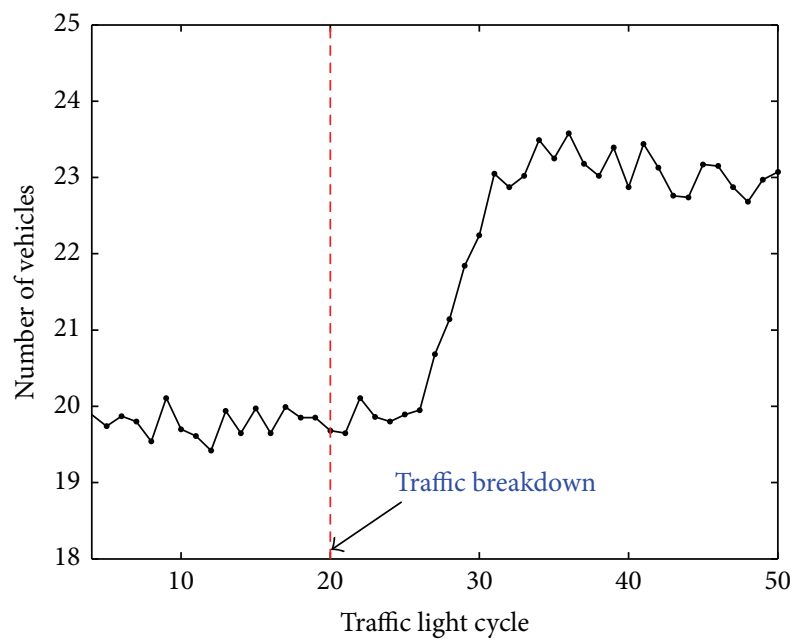

(a)

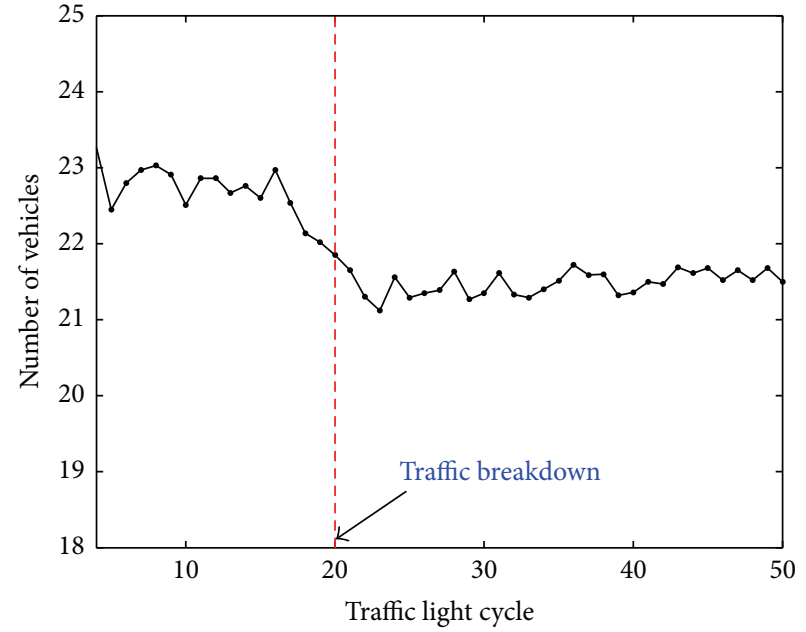

(b)

FIGURE 4: Number of vehicles passing the effective point (a) and the traffic light (b) for each cycle.

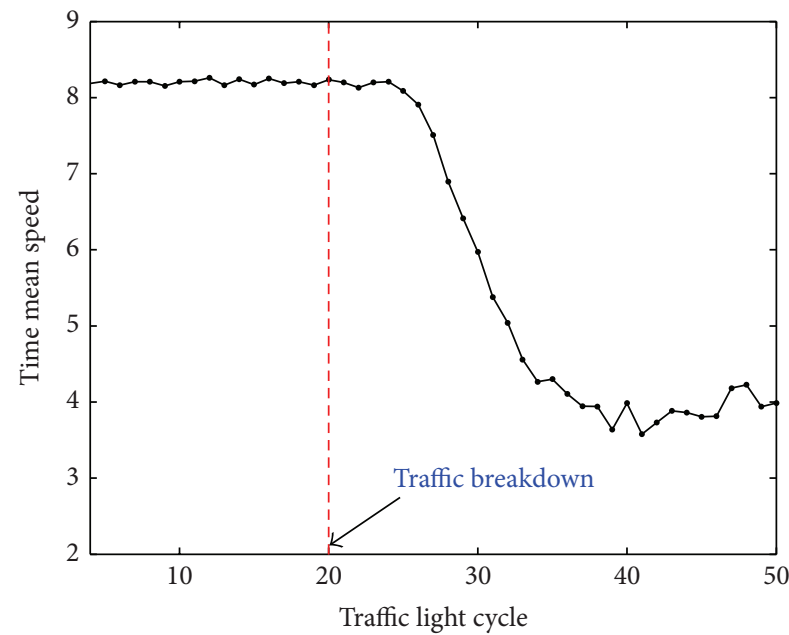

(a)

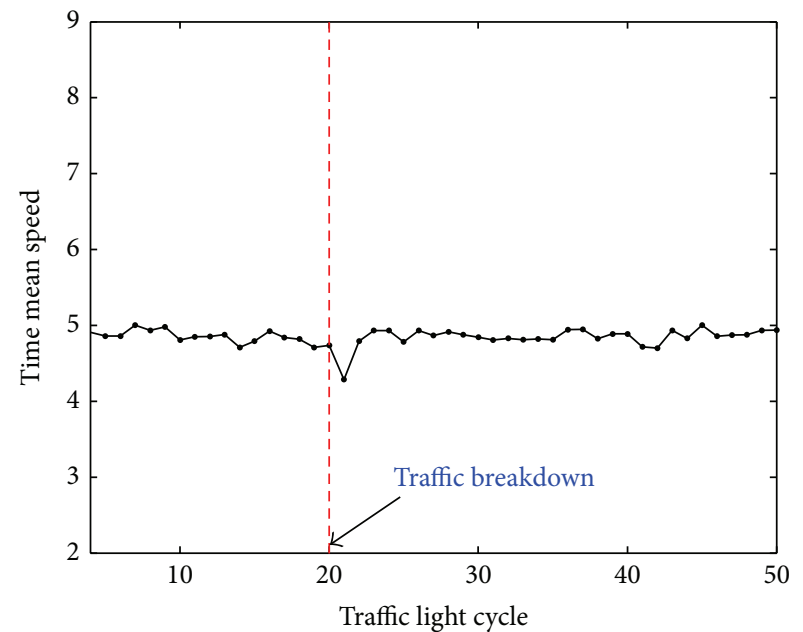

(b)

FIGURE 5: Time mean speed of vehicles passing the effective point (a) and the traffic light (b) for each cycle.

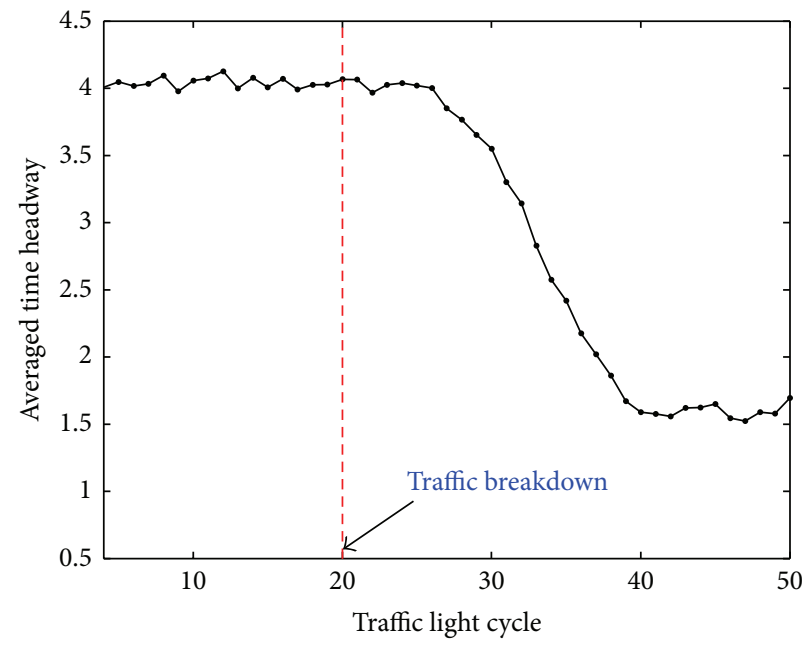

(a)

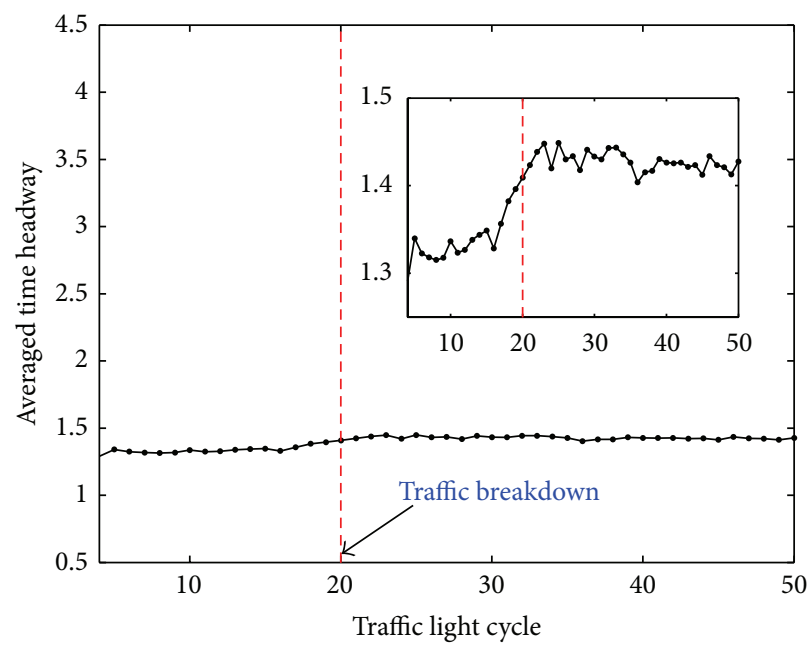

(b)

FIGURE 6: Averaged time headway of vehicles passing the effective point (a) and the traffic light (b) for each cycle. 


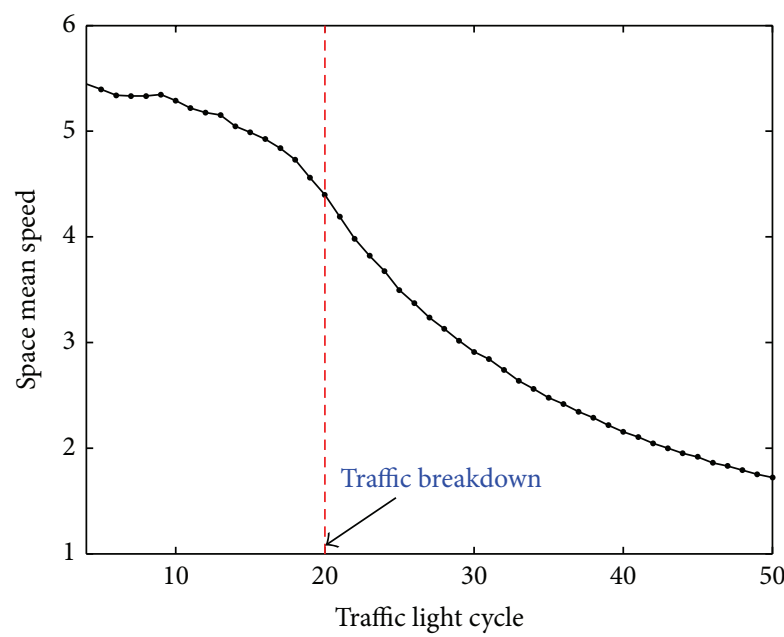

(a)

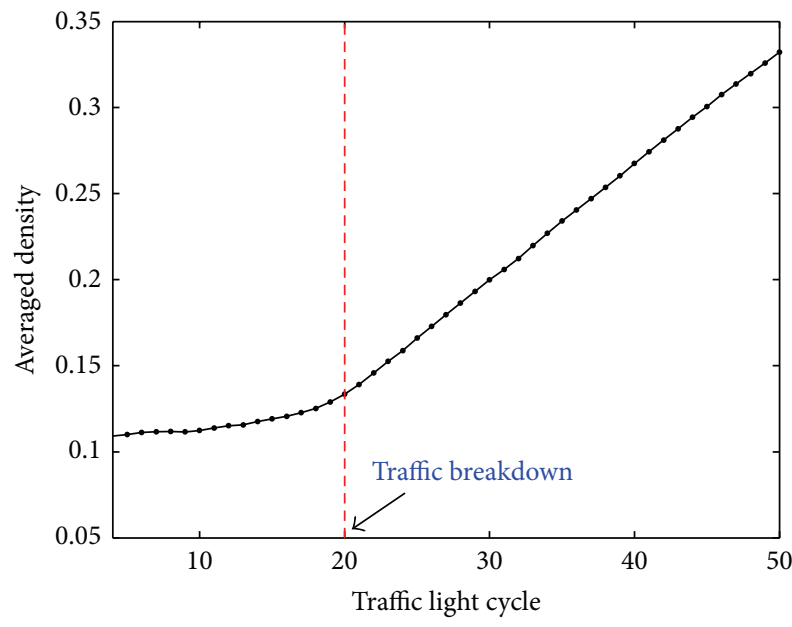

(c)

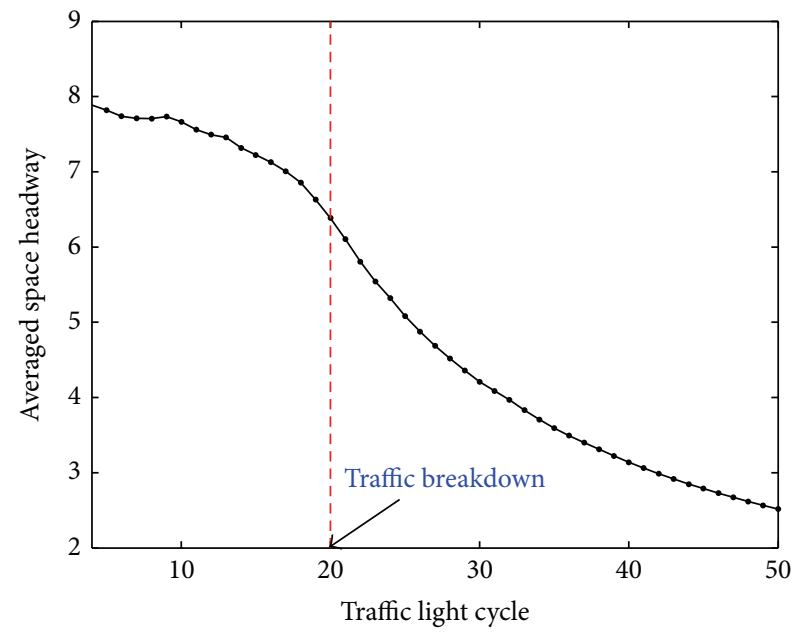

(b)

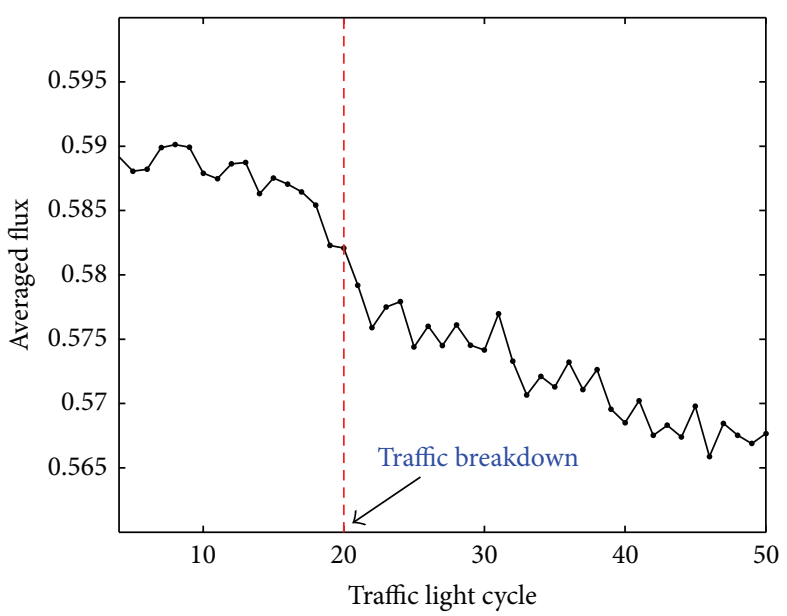

(d)

FIGURE 7: Space mean speed (a), averaged space headway (b), averaged density (c), and averaged flux (d) for the examination of the timedelayed traffic breakdown.

Figure 4(b). The possible reason is that the growth of the queue in front of the traffic light will result in an increase in the probability of the slow-start and consequently extend the stopped time. This is evident by the increase in the averaged time headway shown in the figure inserted in Figure 6(b). The increased time headway will result in the decrease of number of vehicles passing the traffic light as the green phase is fixed.

The results shown in Figure 7 were measured by taking into account the whole road segment rather than a local point as discussed above. As shown in Figures 7(a) and 7(b), the space mean speed and the averaged space headway have very similar patterns. A general decrease can be observed over the test period. The decrease becomes quicker just after the traffic breakdown, but the decrease speed tends to gradually reduce. An opposite pattern is shown in Figure 7(c), implying that the road segment becomes denser after the traffic breakdown. From Figure 7(d), it can be seen that the averaged flux has a downward tendency over the test period.

3.2. Dissolution of the Oversaturated Traffic. In the second set of experiments, we activated the anticipatory behavior after the oversaturated traffic lasted for 5 cycles and the obtained result is shown in Figure 8. Before activating the anticipatory behavior, the traffic experienced a transition from the initial undersaturation to the ove-saturation at the 22 cycle, which was realized using the same setting as that in the previous subsection. It can be seen that the queue starts to shrink after incorporating the anticipatory behavior and the traffic is reversed back to undersaturation since the 33 cycles. Therefore, it is evidenced that the anticipatory behavior can help the queue dissolve.

The results shown in Figures 9 to 12 have been averaged over 100 independent runs with the transition from undersaturation to oversaturation at the 20 cycles and the reversed transition at the 32 cycles approximately.

Figure 9 shows the number of vehicles passing the effective point and the traffic light in each cycle. The number of vehicles shown in Figure 9(a) does not change significantly over the test period. However, Figure 9(b) shows that a slight decrease occurs around the traffic breakdown, but an increase can be observed before the traffic becomes undersaturated 


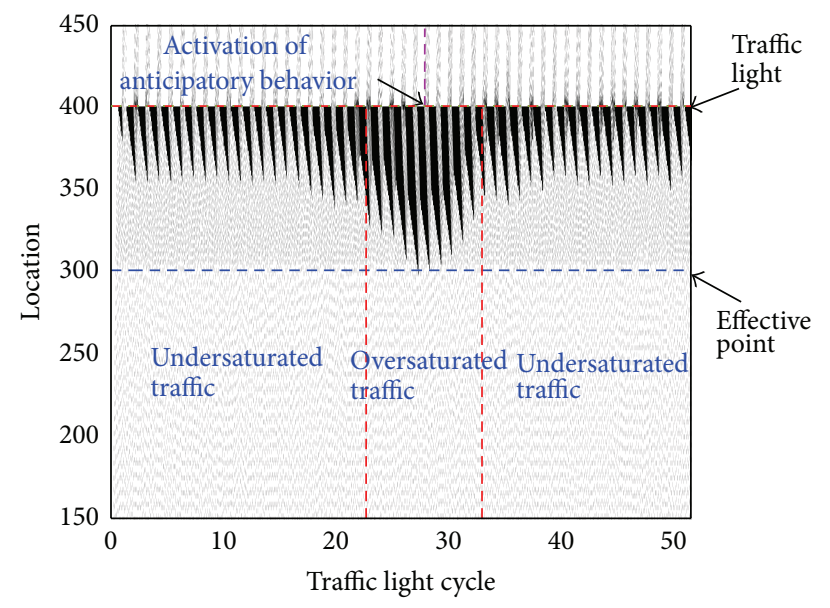

FIGURE 8: Dissolution of the oversaturated traffic after activation of the anticipatory behavior.

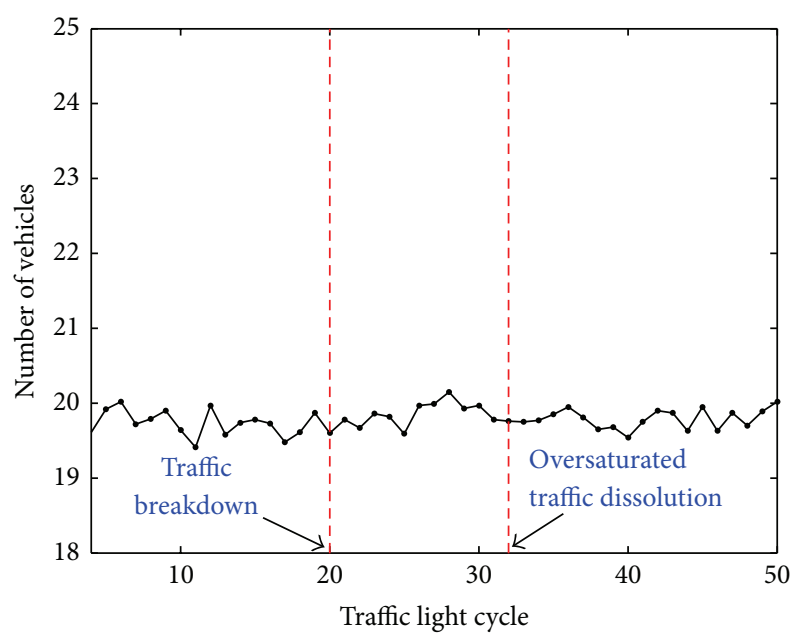

(a)

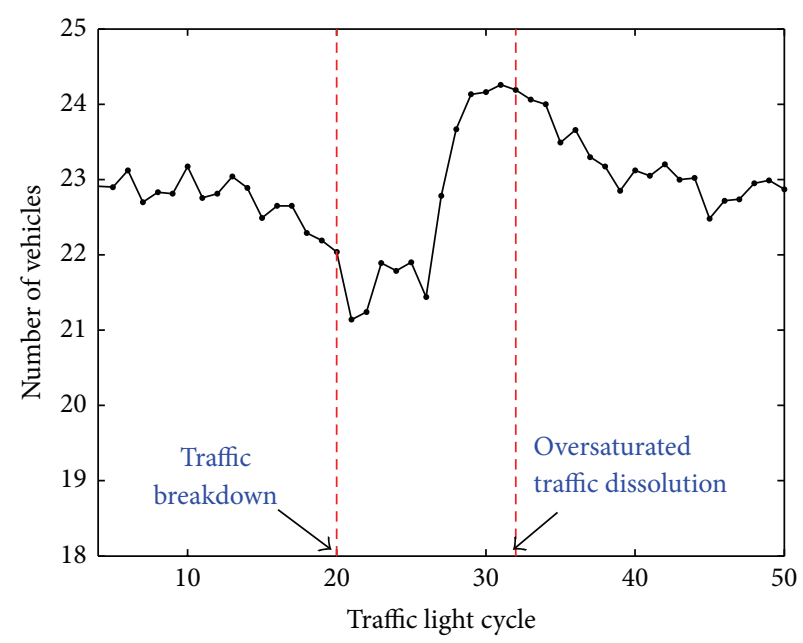

(b)

Figure 9: Number of vehicles passing the effective point (a) and the traffic light (b) for each cycle.

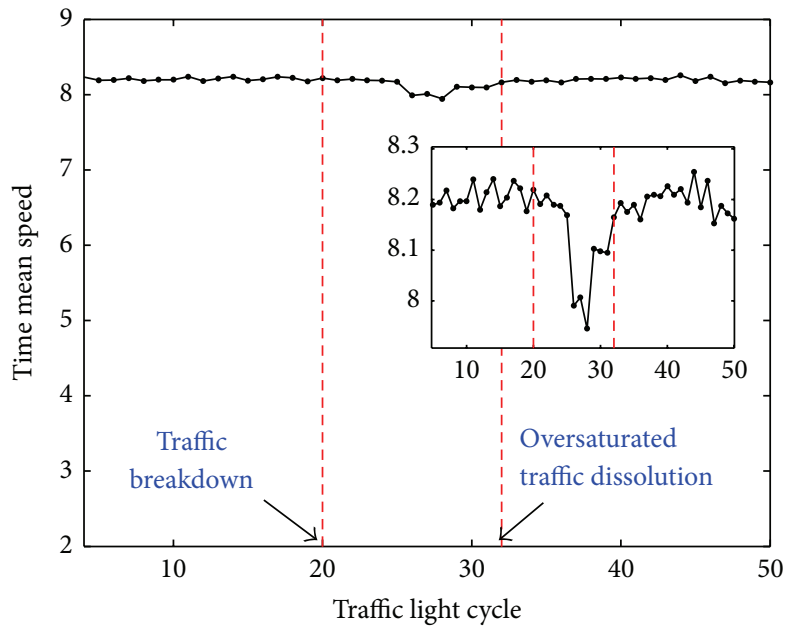

(a)

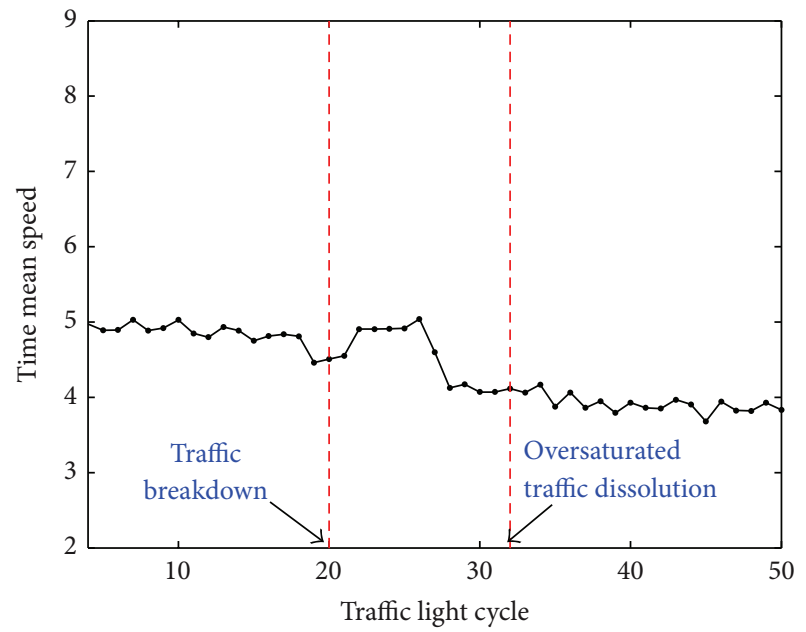

(b)

FIGURE 10: Time mean speed of vehicles passing the effective point (a) and the traffic light (b) for each cycle. 


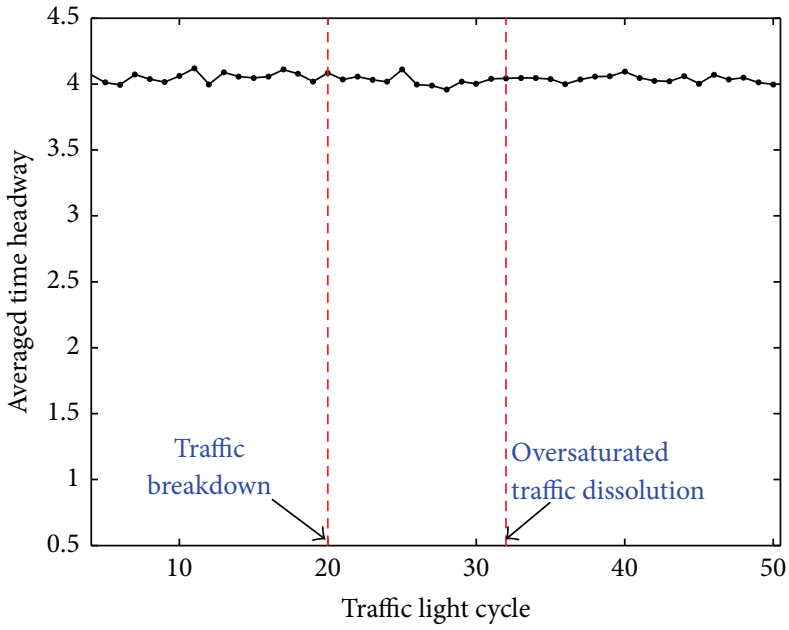

(a)

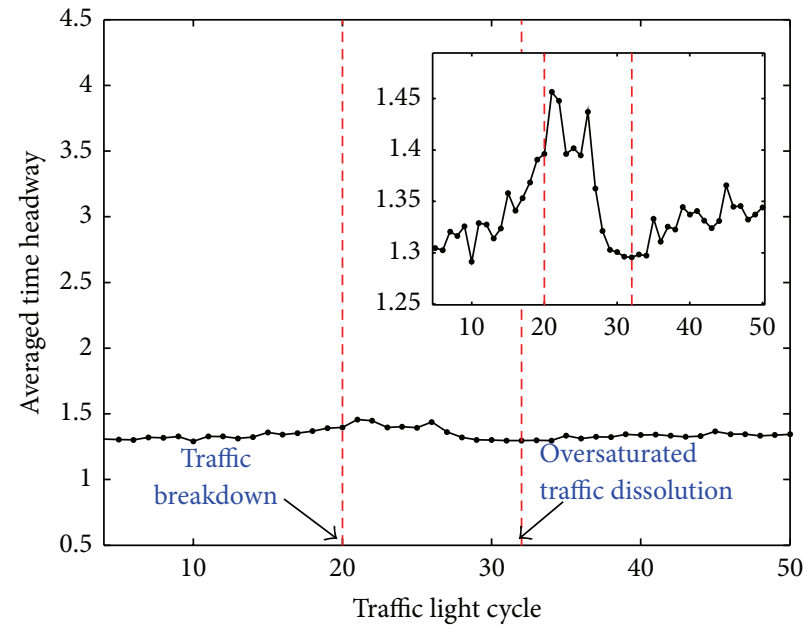

(b)

FIGURE 11: Averaged time headway of vehicles passing the effective point (a) and the traffic light (b) for each cycle.

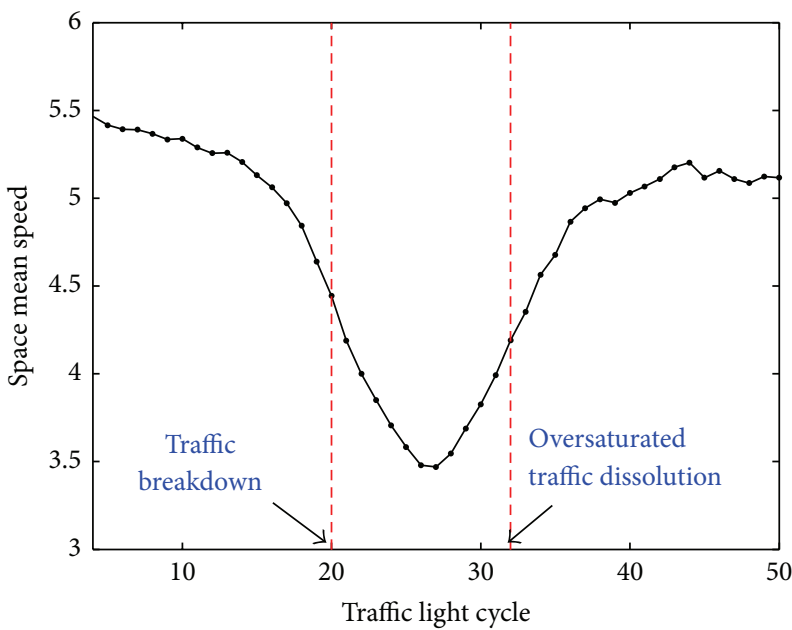

(a)

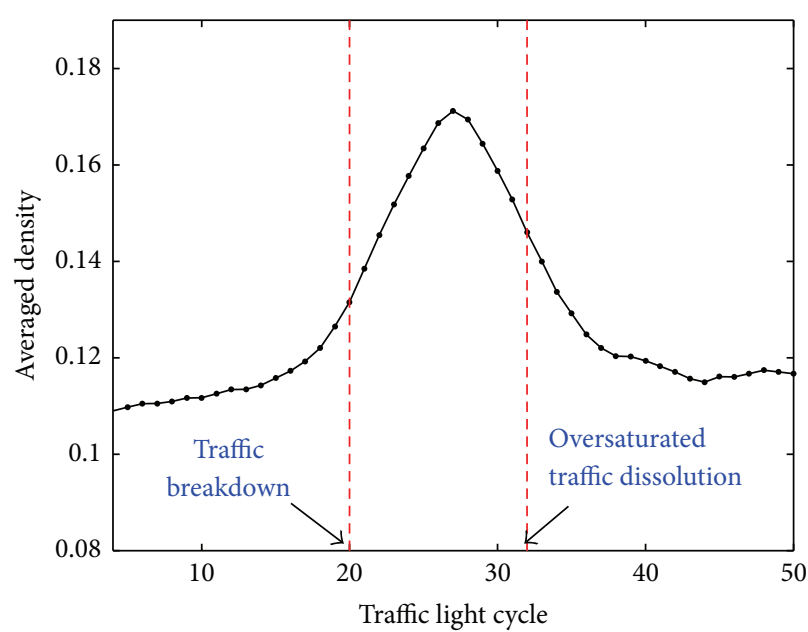

(c)

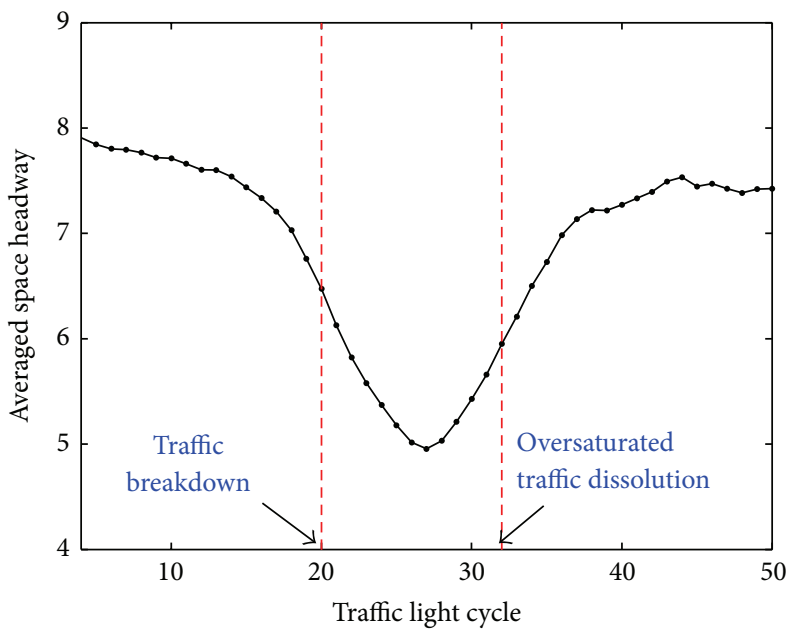

(b)

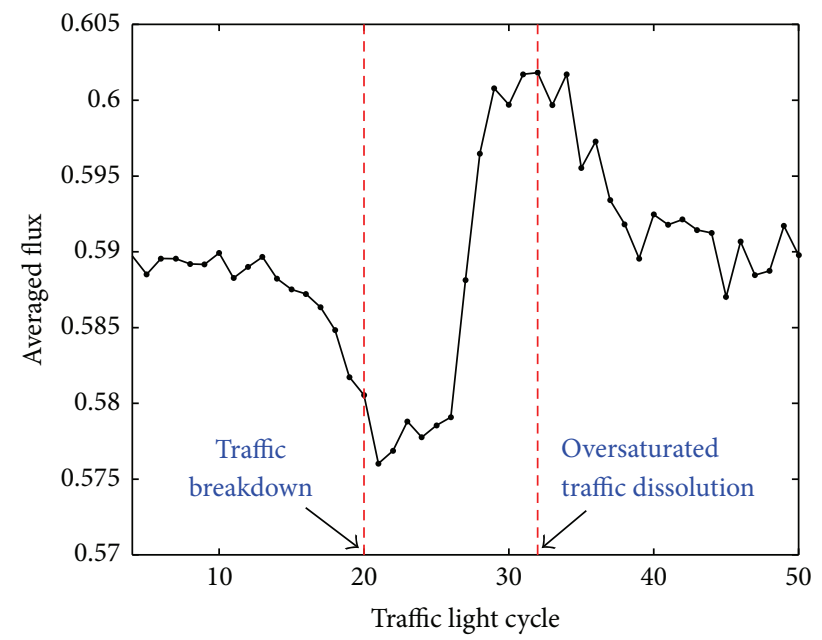

(d)

Figure 12: Space mean speed (a), averaged space headway (b), averaged density (c), and averaged flux (d) for the examination of the anticipatory behavior. 


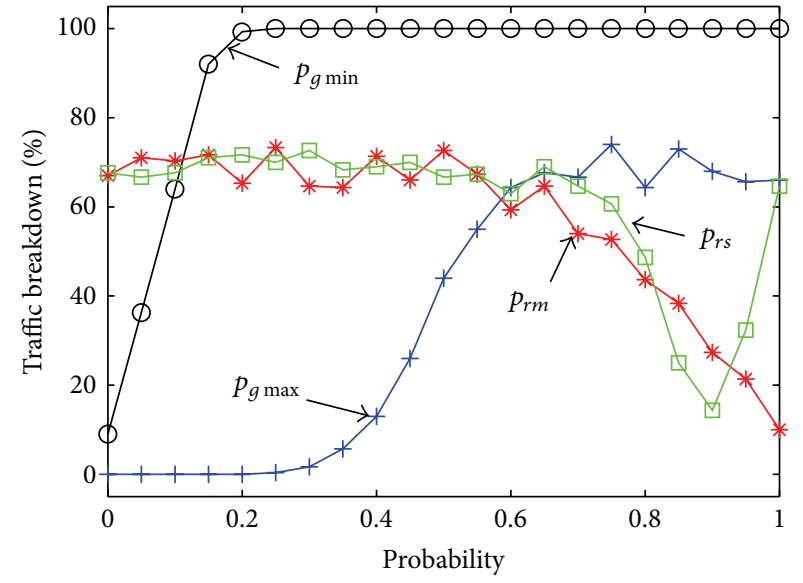

(a)

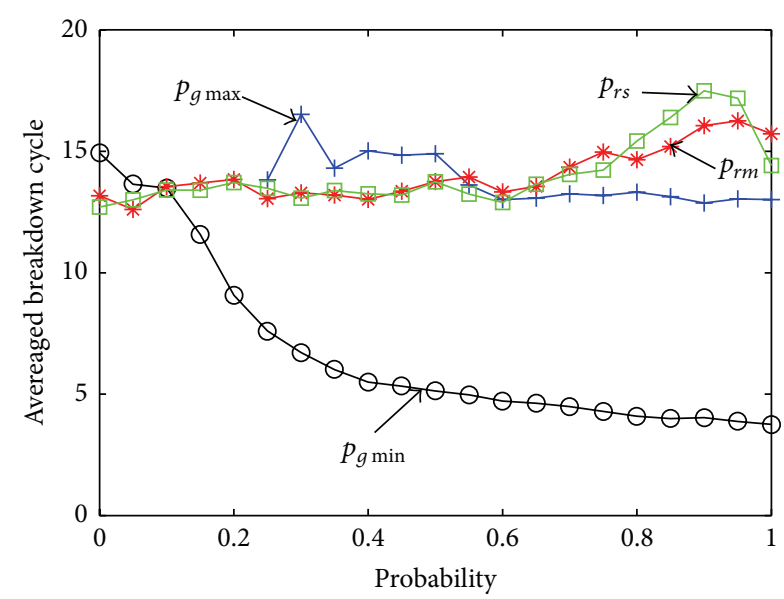

(b)

FIGURE 13: Percentage of traffic breakdown (a) and averaged breakdown cycle (b) for the probabilities $p_{g \text { min }}, p_{g \text { max }}, p_{r m}$, and $p_{r s}$.

again, and a decrease is generally dominated in the cycles since the transition from the oversaturated traffic to the undersaturated traffic.

Figure 10(a) shows that the time mean speeds vary slightly over the test period. The figure inserted in Figure 10(a) has zoomed out the traffic evolution, which shows a small valley around the 28 cycle. However, the pattern shown in Figure 9 (b) is more complex. The time mean speed firstly drops slightly before the traffic breakdown and then increases after the breakdown. A relatively big drop occurs at the 26 cycles and the 27 cycles and a gentle decrease over the rest of the test period.

Figure 11 shows the averaged time headways of the vehicles passing the effective point and the traffic light. As shown in Figure 11(a), there are not significant changes on the averaged time headways over the test period. However, from Figure 11(b), a rise and fall can be observed from the 10 cycles to the 30 cycles approximately.

By considering the whole road segment for each cycle, we have obtained the space mean speed, averaged space headway, averaged density, and averaged flux, as shown in Figures 12(a), 12(b), 12(c), and 12(d), respectively. Although Figures 12(a) and 12 (b) present different parameters, their distributions over the test period are very similar. When the traffic transits from the initial undersaturation into the oversaturation, a drop can be observed in the both figures. Furthermore, these two figures show that the increases start from the identical cycle at which the anticipatory behavior has just taken effect. The increases continue even after the traffic is reversed back to the undersaturation but stop at the level lower than those before the breakdown. By a close observation, it can be found that the result shown in Figure 12(c) seems as if the curve in Figure 12(b) had turned upside down. Also, it is clear that the traffic is much denser during the oversaturation period than that during the undersaturation period. Figure 12(d) shows a sharp decrease around the traffic breakdown and an increases starting at the cycle when the anticipatory behavior was activated and stopping with the maximum value before the flux begins to drop again since the reversed transition.
The increase of the flux can be attributed to the anticipatory behavior as it can result in a relatively large speed even for a small gap. When the queue is dissolved, the flux comes to the similar level as that during the first several cycles.

3.3. The Influence of Driving Behaviors on the Traffic Breakdown. In order to exam the influence of the microscopic behaviors on the traffic breakdown, a number of simulations were carried out by varying the probabilities $p_{g \text { min }}, p_{g \text { max }}$, $p_{r m}$, and $p_{r s}$, respectively, from 0 to 1 with a constant interval of 0.05 . The percentage and the averaged cycle at which the traffic breakdown occurred were estimated by taking the mean value over 300 independent runs and the corresponding results are shown in Figure 13 with the markers "O," "+," “*, and “ $\square$ " for $p_{g \text { min }}, p_{g \text { max }}, p_{r m}$, and $p_{r s}$, respectively. In order to avoid the possible influence of the anticipatory behavior on the traffic breakdown, it was inactivated in this set of experiments.

When examining the slow-to-start behavior, $p_{g \text { min }}$ was firstly varied, but the other probabilities were kept the same as those used to produce the results in Figure 2. From Figure 13(a), it can be seen that the percentage of the traffic breakdown increases rapidly before $p_{g \text { min }}$ reaches 0.2 . When $p_{g \text { min }}$ is greater than 0.2 , the traffic breakdown will always occur. With the increase of $p_{g \text { min }}$, the cycle at which the traffic breakdown occurred gradually decreases from 15 to 4 approximately. Secondly, $p_{g \text { max }}$ was varied, but the others set the same as those stated above. It is apparent that the percentage of the traffic breakdown is an increasing function of $p_{g \text { max }}$, but the averaged breakdown cycle is the opposite. The traffic breakdown does not occur within the test period unless $p_{g \text { max }}$ increases to 0.3 approximately. On the other hand, the transition happens not all times within the test cycles, even if $p_{g \text { max }}$ is equal to 1 . Finally, we examined the influence of the parameter $\theta_{g}$ on the traffic breakdown by varying it from 0 to 0.02 with a fixed interval of 0.005 but the other parameters were kept as the same as the first set of experiments, and the obtained results are shown in Figure 14. The percentage of traffic breakdown approaches to zero when 


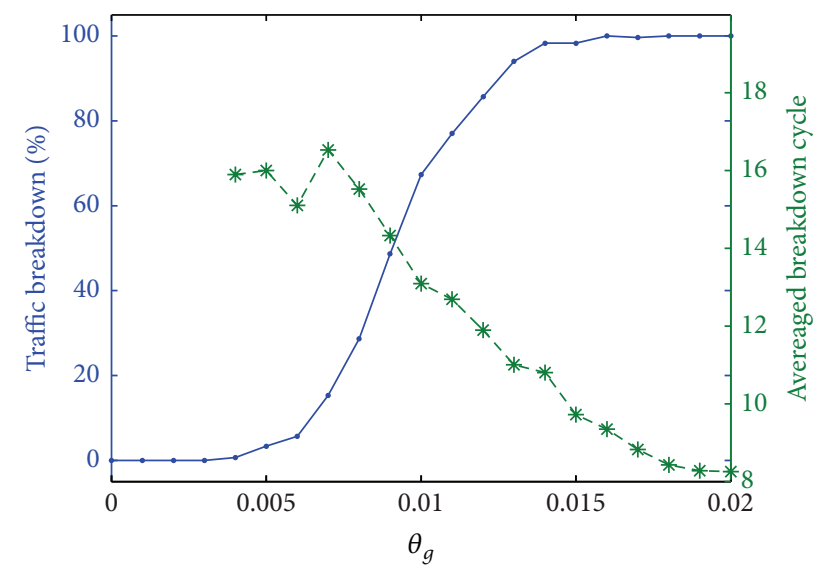

FIGURE 14: Percentage of traffic breakdown (with the marker “•”) and averaged breakdown cycle (with the marker “*”) for different $\theta_{g}$.

$\theta_{g}$ is less than 0.005 , but it quickly increases to $100 \%$ or near when $\theta_{g}$ increases from 0.005 . On the other hand, a general decrease of the averaged breakdown cycle can be seen with the increase of $\theta_{g}$. Note that the traffic breakdown does not occur when $\theta_{g}$ is less than 0.004 . In short, the slow-to-start behavior has significant influence on the traffic breakdown and the slower the stopped vehicles restart, the more likely the traffic breakdown occurs.

Next, we varied $p_{r m}$, which is believed to be the means to control the slowdown behavior, but kept the others as same as those stated at the beginning of Section 3.1. From Figure 13, it can be seen that the percentage of the traffic breakdown decreases but the averaged breakdown cycle increases gradually when $p_{r m}$ becomes large, implying that this behavior negatively affects the traffic breakdown.

Finally, the inch-forward behavior was evaluated by varying $p_{r s}$ but not the others. The percentage of the traffic breakdown fluctuates around $70 \%$ and the breakdown cycle around 13 when $p_{r s}$ is less than 0.7 approximately. Since $p_{r s}$ is greater than 0.7 but less than 0.9 , a decrease in the percentage and an increase in the breakdown cycle can be observed in general, but there appears an opposite changes just before $p_{r s}$ reaches 1 from 0.9 .

\section{Conclusions}

The existing CA models for the signal controlled traffic has considered a few driving behaviors and there is a potential risk that these models may not be able to uncover all possible traffic phenomena. To remedy this defect, the present work has firstly attempted to formulate different rules to reflect the driver's responses to the different signals and then the rules for different signal phases have been unified. Unlike the previous works, the signalized road has been segmented into three sections in that the signal influence on driving is not unique and different rules have been developed for the sections.

Based on the numerical simulations, the followings can be concluded:

(1) the proposed CA model can produce the time-delayed traffic breakdown and the dissolution of the oversaturated traffic;
(2) the driving behaviors that can potentially result in the macroscopic traffic phenomenon are identified and, particularly, we have found that the anticipatory behavior can efficiently promote the dissolution of the oversaturated traffic;

(3) the influences of driving behaviors on the traffic breakdown are quantitatively evaluated.

Nevertheless, only the primary investigation on the proposed model is presented in this paper and a more extensive exploration on the model dynamics will be conducted in the next stage.

\section{Conflict of Interests}

The authors declare that there is no conflict of interests regarding the publication of this paper.

\section{Acknowledgments}

This paper is financially supported by the National Basic Research Program of China (Grand no. 2012CB723303) and the Beijing Committee of Science and Technology, China (Grand no. Z1211000003120100).

\section{References}

[1] K. Nagel and M. Schreckenberg, "A cellular automaton model for freeway traffic," Journal de Physique I, vol. 2, no. 12, pp. 22212229, 1992.

[2] Y. Ishibashi and M. Fukui, "Temporal variations of traffic flow in the Biham-Middleton-Levine model," Journal of the Physical Society of Japan, vol. 63, no. 8, pp. 2882-2885, 1994.

[3] R. Barlovic, L. Santen, A. Schadschneider, and M. Schreckenberg, "Metastable states in cellular automata for traffic flow," European Physical Journal B, vol. 5, no. 3, pp. 793-800, 1998.

[4] F. Knorr and M. Schreckenberg, "The comfortable driving model revisited: traffic phases and phase transitions," Journal of Statistical Mechanics: Theory and Experiment, vol. 2013, no. 7, Article ID P07002, 2013.

[5] M. E. Larraga, J. A. del Rio, and A. Schadschneider, "New kind of phase separation in a CA traffic model with anticipation," Journal of Physics A: Mathematical and General, vol. 37, no. 12, pp. 3769-3781, 2004.

[6] R. Jiang and Q.-S. Wu, "Open boundaries in a cellular automata model for synchronized flow: effects of nonmonotonicity," Physical Review E, vol. 68, no. 2, Article ID 026135, 2003.

[7] J.-F. Tian, B. Jia, X.-G. Li, R. Jiang, X.-M. Zhao, and Z.-Y. Gao, "Synchronized traffic flow simulating with cellular automata model," Physica A: Statistical Mechanics and its Applications, vol. 388, no. 23, pp. 4827-4837, 2009.

[8] Z.-T. Xiang, Y.-J. Li, Y.-F. Chen, and L. Xiong, "Simulating synchronized traffic flow and wide moving jam based on the brake light rule," Physica A: Statistical Mechanics and Its Applications, vol. 392, no. 21, pp. 5399-5413, 2013.

[9] J.-F. Tian, N. Jia, N. Zhu, B. Jia, and Z.-Z. Yuan, "Brake light cellular automaton model with advanced randomization for traffic breakdown," Transportation Research Part C: Emerging Technologies, vol. 44, pp. 282-298, 2014. 
[10] K. Gao, R. Jiang, B.-H. Wang, and Q.-S. Wu, "Discontinuous transition from free flow to synchronized flow induced by shortrange interaction between vehicles in a three-phase traffic flow model," Physica A, vol. 388, no. 15-16, pp. 3233-3243, 2009.

[11] B. Jiaa, X.-G. Lia, T. Chenb, R. Jiangc, and Z.-Y. Gao, "Cellular automaton model with time gap dependent randomisation under Kerner's three-phase traffic theory," Transportmetrica, vol. 7, no. 2, pp. 127-140, 2011.

[12] J. P. L. Neto, M. L. Lyra, and C. R. da Silva, "Phase coexistence induced by a defensive reaction in a cellular automaton traffic flow model," Physica A, vol. 390, no. 20, pp. 3558-3565, 2011.

[13] A. Schadschneider, D. Chowdhury, and K. Nishinari, Stochastic Transport in Complex Systems: From Molecules to Vehicles, Elsevier Science, Oxford, UK, 2010.

[14] D. W. Huang and W. N. Huang, "Traffic signal synchronization," Physical Review E, vol. 67, no. 3, Article ID 056124, 2003.

[15] T. Neumann and P. Wagner, "Delay times in a cellular traffic flow model for road sections with periodic outflow," The European Physical Journal B, vol. 63, no. 2, pp. 255-264, 2008.

[16] R. Jiang and Q.-S. Wu, "A stopped time dependent randomization cellular automata model for traffic flow controlled by traffic light," Physica A: Statistical Mechanics and its Applications, vol. 364, pp. 493-496, 2006.

[17] A. Varas, M. D. Cornejo, B. A. Toledo et al., "Resonance, criticality, and emergence in city traffic investigated in cellular automaton models," Physical Review E-Statistical, Nonlinear, and Soft Matter Physics, vol. 80, no. 5, Article ID 056108, 2009.

[18] L.-J. Tian, H.-J. Huang, and T.-L. Liu, "Information feedback strategies in a signal controlled network with overlapped routes," Chinese Physics Letters, vol. 26, no. 7, Article ID 078903, 2009.

[19] J. de Gier, T. M. Garoni, and O. Rojas, "Traffic flow on realistic road networks with adaptive traffic lights," Journal of Statistical Mechanics, vol. 2011, no. 4, Article ID P04008, 2011.

[20] D. Chowdhury and A. Schadschneider, "Self-organization of traffic jams in cities: effects of stochastic dynamics and signal periods," Physical Review E, vol. 59, no. 2, Article ID R1311, 1999.

[21] B. S. Kerner, S. L. Klenov, G. Hermanns, P. Hemmerle, H. Rehborn, and M. Schreckenberg, "Synchronized flow in oversaturated city traffic," Physical Review E, vol. 88, no. 5, Article ID 054801, 2013.

[22] B. S. Kerner, S. L. Klenov, and M. Schreckenberg, "Traffic breakdown at a signal: classical theory versus the three-phase theory of city traffic," Journal of Statistical Mechanics, vol. 2014, no. 3, Article ID P03001, 2014.

[23] K. R. Boff and J. E. Lincoln, Engineering Data Compendium, USAF, H. G. Armstrong Medical Research Laboratory, WrightPatterson AFB, Ohio, USA, 1988.

[24] J.-F. Tian, Z.-Z. Yuan, M. Treiber, B. Jia, and W.-Y. Zhang, "Cellular automaton model within the fundamental-diagram approach reproducing some findings of the three-phase theory," Physica A: Statistical Mechanics and its Applications, vol. 391, no. 11, pp. 3129-3139, 2012.

[25] L. Zheng, S. Ma, and S. Zhong, "Analysis of honk effect on the traffic flow in a cellular automaton model," Physica A, vol. 390, no. 6, pp. 1072-1084, 2011.

[26] R. Jiang, M.-B. Hu, B. Jia, and Z.-Y. Gao, "A new mechanism for metastability of under-saturated traffic responsible for timedelayed traffic breakdown at the signal," Computer Physics Communications, vol. 185, no. 5, pp. 1439-1442, 2014. 


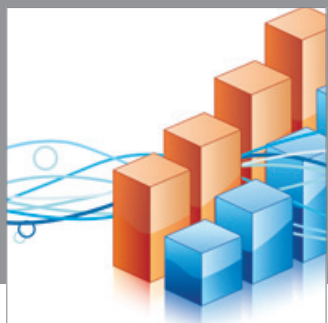

Advances in

Operations Research

mansans

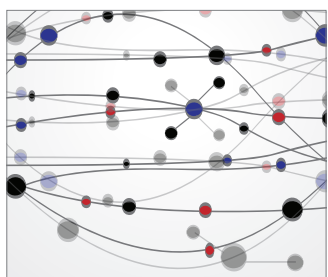

The Scientific World Journal
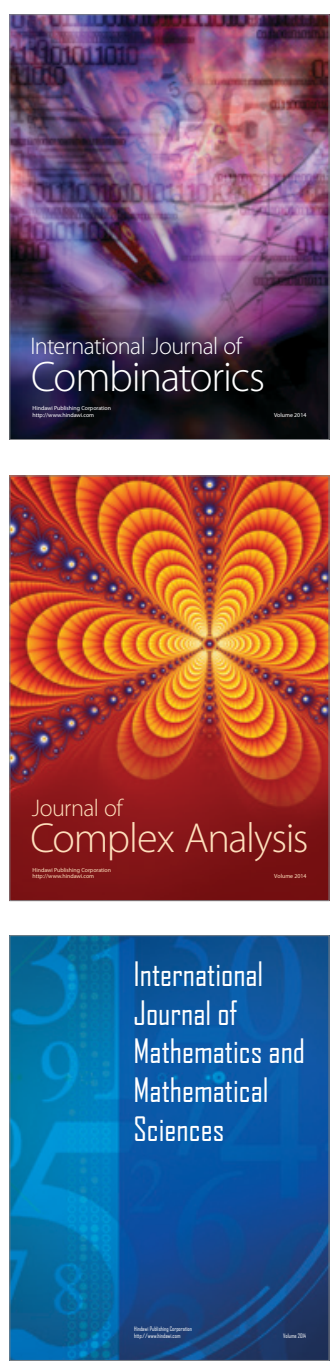
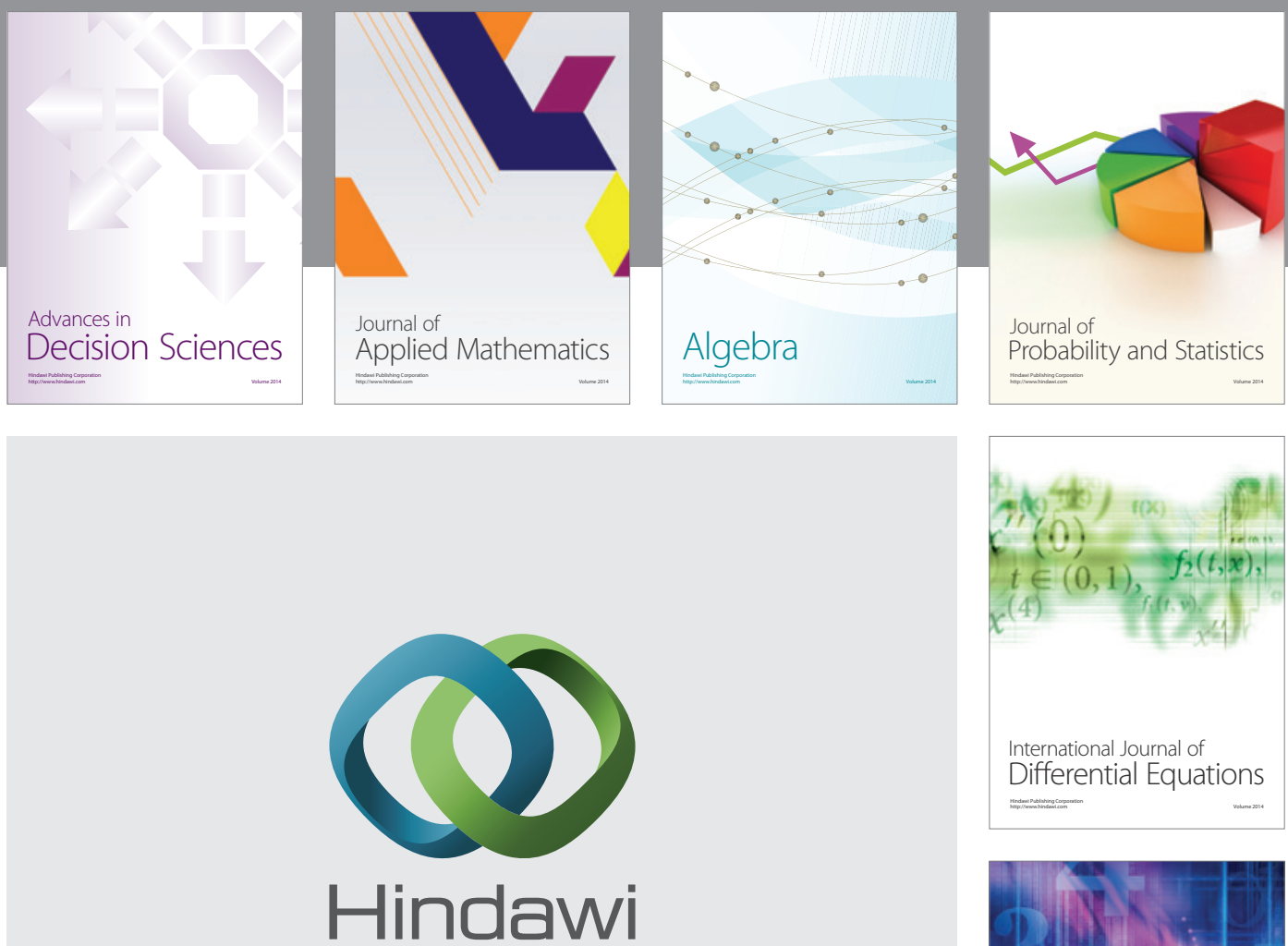

Submit your manuscripts at http://www.hindawi.com
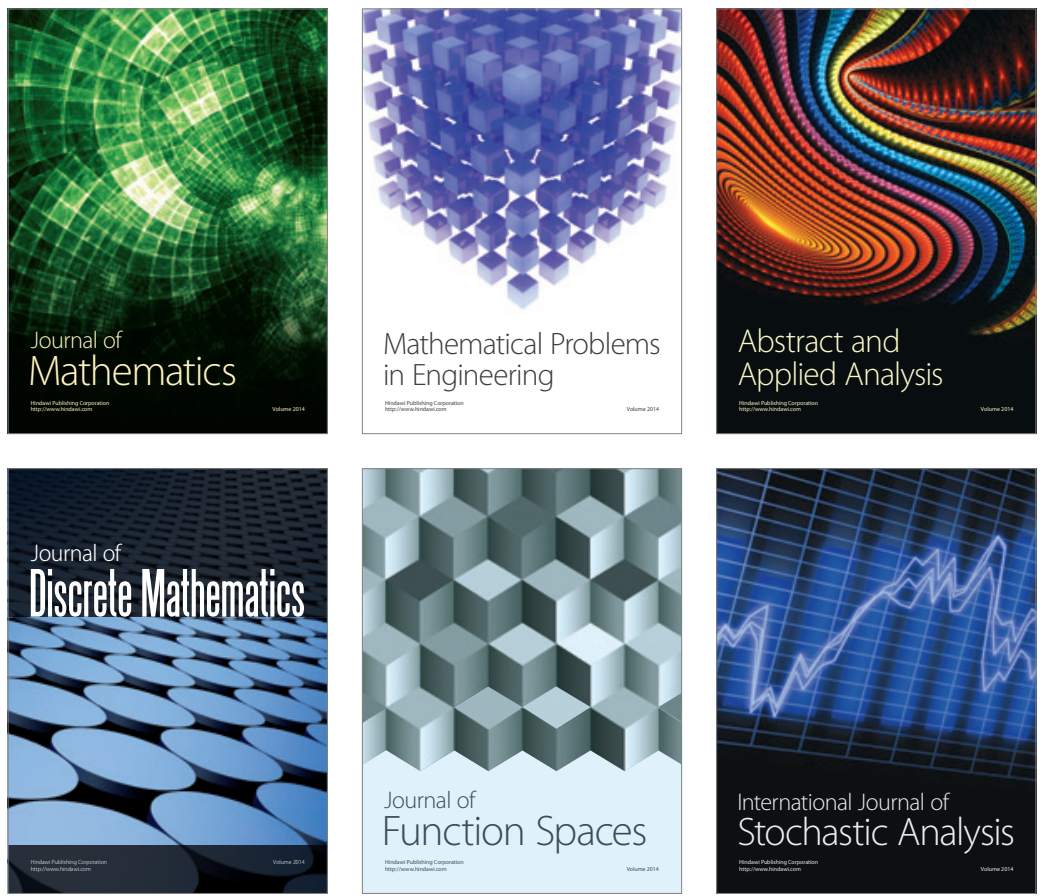

Journal of

Function Spaces

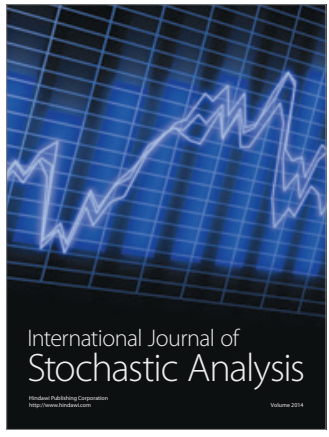

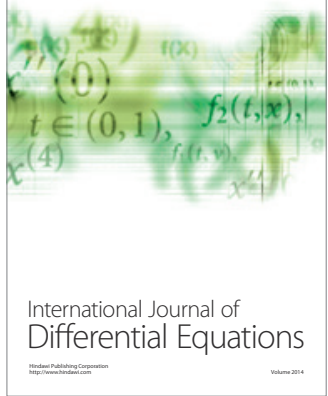
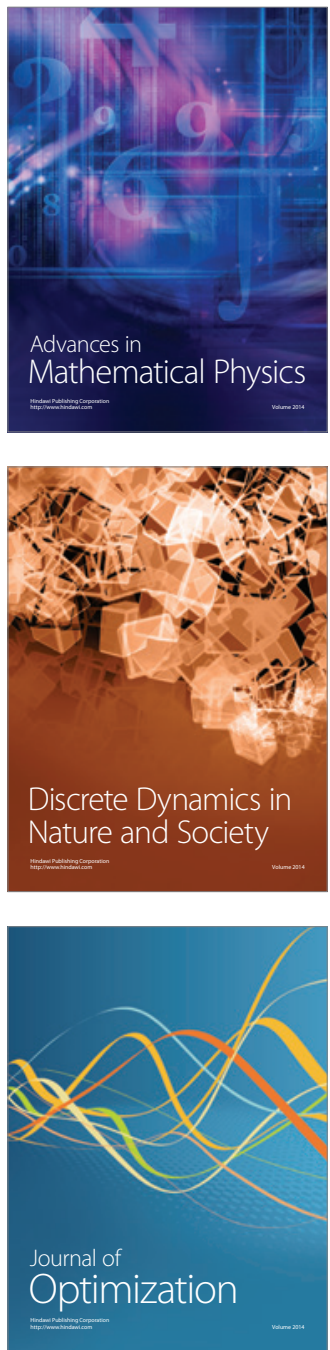